\title{
How to increase awareness of additional vaccinations; the case of maternal pertussis vaccination
}

\author{
K. van Zoonen ${ }^{*}$, W. L. M. Ruijs, H. E. De Melker, M. E. J. Bongers and L. Mollema
}

\begin{abstract}
Background: Effective and safe vaccines are available outside national immunization programs (NIP). Increased awareness and vaccine uptake can improve public health. Before the inclusion of maternal pertussis vaccination (MPV) in the Dutch NIP in December 2019, extra communication efforts were undertaken. Here we examine the success of these efforts, investigating women's awareness of and their decision-making process regarding MPV.

Methods: Between December 2018 and January 2019, one year before the introduction of MPV in the NIP, and about three years after MPV was recommended by the Dutch Health Council, pregnant and non-pregnant women (i.e. child younger than two years) were invited to fill out an online questionnaire. Participant's decision-making processes regarding MPV were assessed with an adapted Precaution Adoption Process Model (PAPM), including stages of awareness, engagement, information-seeking, and vaccination behaviour. Furthermore, factors related to the decision-making process were examined.

Results: In total, 942 women were included, of whom 62\% were non-pregnant. Most of the pregnant and nonpregnant women were aware of MPV during pregnancy (respectively 69 and 56\%). Most aware women had heard about MPV through their midwife and the Public Health Institute (PHI) website. Women unaware of MPV reported a need for information, preferably from their midwives. Most aware women felt MPV was important to them (88\%) and were classified as "engaged". Of the eligible and "engaged" pregnant women, 58\% were vaccinated, versus $38 \%$ of "engaged" non-pregnant women.

Conclusions: As the most preferred and trusted source of information, midwives are essential to increasing awareness of MPV. The PHI website is considered to be a reliable information source and is often consulted. To increase awareness, appropriate healthcare workers should be encouraged to actively inform target groups about available, additional vaccinations.
\end{abstract}

\section{Background}

Comprehensive routine vaccination programmes are in place in many countries worldwide [1]. However, there are additional, effective, and safe vaccines available that are not yet recommended or included in these programmes. In the Netherlands, the National Immunization Program (NIP) offers vaccines free of

\footnotetext{
* Correspondence: Kim.van.zoonen@rivm.nl

Centre for Infectious Disease Control, National Institute for Public Health and the Environment (RIVM), PO Box 1, 3720 BA Bilthoven, The Netherlands
}

charge, and on a voluntary basis. Additional vaccines outside of the NIP are available to the public through out-of-pocket purchases. Information regarding these additional vaccines is provided on the website of the Dutch public health institute (PHI). Additional vaccines are not actively promoted, and consequently both public and health professional awareness is low. With increased awareness, and subsequently increased uptake, of vaccines outside of the NIP there are improvements in public health to be gained [2]. 
Awareness, information seeking, and engagement are essential to the vaccination decision-making process. The process can be explained within the framework of the Precaution Adoption Process Model (PAPM). The model demonstrates how risk mediation mechanisms form pathways to health-protective behaviours, such as vaccination $[3,4]$. The PAPM consists of seven stages from "unaware" to "action" to "maintenance" of healthprotective behaviours. The model also identifies factors (such as barriers to health care, self-efficacy, trust in information sources, decision-making styles, educational level, attitude, and intention) that facilitate or impede movement through the seven stages [3, 5-7].

Since 2015 the Dutch Health Council has recommended a Diphtheria, Tetanus, and acellular Pertussis (dTaP) vaccine for pregnant women, available as an outof-pocket purchase. In December 2019, the maternal pertussis vaccination (MPV) was included as part of the NIP, for administration between 28 to 36 weeks of pregnancy [8]. Between 2015 and 2019, there were different routes for pregnant women to obtain the NIP provided MPV in the Netherlands. In some regions, women had to consult with their general practitioner (GP) to obtain a prescription for the MPV for collection at the local pharmacy. In other regions, women could contact municipal public health services (PHS) directly to receive their vaccine. If a mother is vaccinated during pregnancy her infant would follow a 3, 5, 11-month vaccination schedule, as opposed to the $2,3,5,11$-month vaccination schedule for infants of unvaccinated mothers (see: Dutch National Immunisation Programme Rijksvaccinatieprogramma.nl).

In 2017, prompted by the media attention surrounding the MPV, and following the advice of the Dutch Health Council, the Dutch PHI decided to undertake supplementary communication efforts to increase MPV awareness. Communication efforts were directed at pregnant women and their antenatal care providers. In the Netherlands antenatal care is the responsibility of midwives and/or gynaecologists, and not GPs. Furthermore, GPs are not responsible for administering NIP vaccinations, which are instead delivered by youth health workers (YHW). As such, from 2017 flyers and letters containing MPV information were delivered directly to pregnant women by midwives and gynaecologists. In 2018 further flyers, factsheets and posters were distributed amongst professionals as well as for display in the waiting rooms of primary care services and youth health worker's offices (i.e. PHS).

In this study, we firstly aim to examine the awareness of women regarding MPV, to investigate the success of the 2017/2018 MPV communication efforts. Secondly, we analyse the decision-making process of pregnant women concerning the uptake of the MPV. We aim to identify potential demographic and socio-cognitive factors that may mediate each stage of the MPV uptake decision-making process, within the framework of the PAPM. The results of this study can contribute to improving national, and international, communication efforts to increase awareness and vaccine uptake of nonNIP vaccines.

\section{Methods}

\section{Study population and recruitment}

Between December 2018 and January 2019, women between 18 to 49 years of age were invited to fill out an online survey (see supplementary materials). Invitees were women who were pregnant at the time of the study and women who had been recently pregnant. Recent pregnancy was defined based on the age of the youngest child and was limited to 2 years of age to minimise recall bias. This age limit was also chosen as active MPVrelated information materials (e.g. website, fora, and magazines focusing on pregnant women) were available from 2017 onwards - two years before the study. Additionally, the distribution of information flyers and factsheets at GPs and YHWs offices began in 2018. Women were directly recruited through an online survey panel (Flycatcher, ISO 26362, https://www.flycatcherpanel.nl/ nld/over-het-panel/). Participants also recruited other women not included in the original sampling frame, a method known as snowball sampling. All participants provided active consent irrespective of their recruitment route. All survey questions related to the current, or the most recent, pregnancy of the participant.

This study was not subject to the law in the Netherlands regarding medical research involving human subjects according to The Clinical Expertise Centre RIVM (No. LCI-423). Therefore, this study was exempt from requiring further approval from an ethics research committee.

\section{The precaution adaptation model (PAPM)}

The PAPM was used as a theoretical framework to develop the survey. We adapted the model to investigate two behaviours pertinent to MPV awareness and uptake: information seeking and vaccination behaviour (Fig. 1). Stage 1 of the adapted model examines the awareness of the MPV. In Stage 2 women engage with the decisionmaking process of whether or not the MPV is important or relevant to them. Stage 2 can only be entered once women become aware of the MPV, in other words, once women have entered Stage 1 [3]. After Stage 2, women can enter Stage 2B and search for (additional) information to support the decision-making process, and subsequently enter Stage 3, at which point the decision to vaccinate or not is made. Women can also bypass Stage 2B and enter Stage 3 directly from Stage 2 (Fig. 1). Stage 


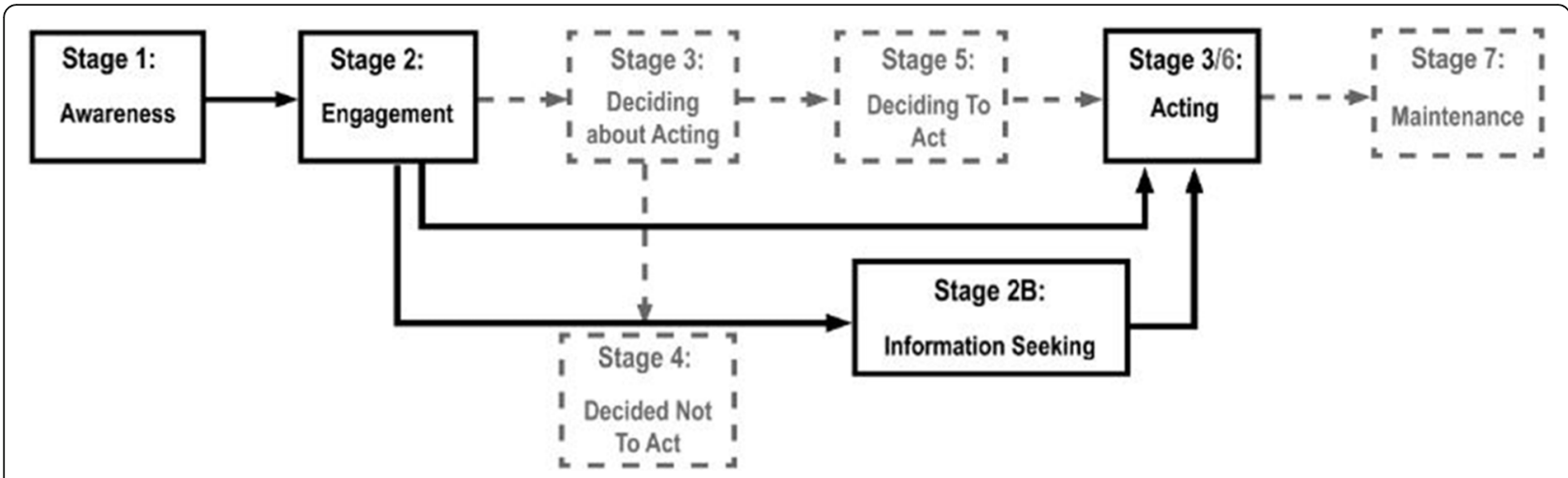

Fig. 1 Original complete PAPM (black and greyscale boxes and arrows) and the model adapted from the PAPM for this study (black boxes and arrows only)

2B and Stage 3 can only be entered after women have engaged with the MPV in Stage 2. Stages $2 B$ and 3 of the adapted model replaced Stage 3 through to 6 of the original model and sage 7 of the original PAPM was excluded as vaccination with the MPV is not a recurrent or maintained behaviour (Fig. 1).

\section{Survey}

A pilot survey was conducted to optimize the final questionnaire and ensure the questions and answers were comprehensive and logical. Questions regarding demographics (e.g. age, education, ethnicity), awareness of the MPV, information delivery preferences (i.e. if women were unaware, would they have preferred to hear or read about MPV), engagement with the MPV, MPV information-seeking behaviour, and vaccination uptake were included in the final questionnaire. In addition, the survey included questions regarding factors that may influence the individual stages of the adapted PAPM. For Stage 1 questions examined MPV information sources, formats, and individual preference for information format. In Stage 2, Stage 2B, and Stage 3 questions assessed participant critical thinking (i.e. the ability to distinguish different information about MPV and take into account the reliability of an information source), descriptive norm (i.e. most women will get vaccinated during their pregnancy) and participant trust in three information sources (midwives, PHI and PHS). Stage $2 \mathrm{~b}$ and Stage 3 included further questions regarding participant selfefficacy, injunctive norms, considerations (i.e. possible barriers or facilitators). For Stage $2 \mathrm{~B}$ the duration of search for information, information sources, trust in information sources, and subjective knowledge of the MPV was examined. For Stage 3, attitudes, intentions, information-seeking behaviours, and vaccine provider were examined. This section of the survey also explored reasons for deciding about MPV. This was done using 6 subjective consideration statements. Three subgroups of statements investigated reasons not to vaccinate. The first group of statements related to possible advantages for the participant's baby, and included the statements "If my baby would need one vaccination less, I would get MPV" and "If the baby would get its first vaccinations later in life, I would get MPV". The second group of statements considered the route of MPV administration, and included the statements "I would get the vaccination if it is provided by my midwife", "If MPV is provided by someone other than my GP or midwife I would not go", and "If MPV is included in the NIP I would get it". The final group of statements related to the accessibility for obtaining MPV, and included the statement "If I wanted to get the MPV I would know what to do".

For both Stage 2B and Stage 3 decision-making styles were investigated $[9,10]$. To identify the decisionmaking styles of the participants the Dutch version of the General Decision Making Style (GDMS) was included in the survey. It consists of 25 items corresponding to five decision making styles; i) a rational style with emphasis on a thorough and logical process, ii) intuitive style with emphasis on intuition and inner feelings, iii) dependent style with emphasis on relying on the advice of others, iv) avoidant style with emphasis on avoiding making a decision, and v) impulsive (or spontaneous) style with emphasis on making a decision as soon as possible [11, 12]. These styles are independent, but not mutually exclusive [13].

\section{Statistical analyses}

Firstly, for each survey item, the Cronbach's alpha $(\alpha)$ was identified, and scales were adjusted to ensure each survey item was summed to one scale (see Appendix Table 1). Survey items relating to injunctive norms were analysed separately due to their relatively low Cronbach's alpha (0.66). Within the subjective considerations question panel the survey item "If MPV is provided by someone other than my GP or midwife I would not go" 
was recoded to match the scale of the other subjective consideration statements.

Secondly, MPV information materials (e.g. website, fora, and magazines focusing on pregnant women) became available in 2017, and additional information flyers and factsheets were distributed from 2018. As such, non-pregnant women probably had less access to certain relevant information materials. Therefore, analyses per PAPM stage were conducted separately for pregnant and non-pregnant women. All data analyses were performed in SPSS for Windows (version 24.0). Chi-squared tests, independent sample t-tests, and ANOVA's were conducted. If cell counts were low, the categories were recoded, and the Welch test was examined when Levene's test was significant.

\section{Results}

\section{Overall}

In total, 947 women completed the questionnaire. 5 women were excluded as they fell outside of the age range (18-49 years) [14]. Therefore, the net survey sample was 942 women (Table 1).

The average age of participants was 31.8 years. The majority of participants were highly educated (67\%) and most were of Dutch nationality (94\%). Most women completed the questionnaire through Flycatcher (84\%). The majority of women were non-pregnant $(62 \%)$. Nonpregnant women were on average older than pregnant women ( 32.4 years vs 30.8 years respectively). Of the 358 pregnant women, nearly $60 \%$ already had an older child. The average gestational age of pregnant participants was 21 weeks (range 2-42 weeks). When comparing nonpregnant women to pregnant women, pregnant women were more frequently aware of the MPV $(69.3 \%$ vs $56.2 \%, N=942$ ), sought information about the MPV (44.7\% vs $36.8 \%, \mathrm{~N}=942)$ and were more often vaccinated with the MPV ( $31 \%$ vs $19.9 \%, \mathrm{~N}=942)$. The percentages of neutral and engaged pregnant women were similar to the percentages of neutral and engaged nonpregnant women ( $86.3 \%$ vs $83.2 \%$ respectively, $\mathrm{N}=942)$.

\section{Stage 1: awareness of MPV}

Approximately $69 \%$ of pregnant women and $56 \%$ of non-pregnant women were aware of MPV (Table 2). Of those, most women had heard about MPV through their midwife (49\% of pregnant women and 39\% of nonpregnant women). However, pregnant women also reported that other health care workers $(\mathrm{HCW})$, such as GPs or PHS, had been their MPV information sources. Non-pregnant women reported the PHI website was an important information source. Non-pregnant women reported they would prefer to be informed about the MPV through the internet (35\%), whereas pregnant women reported preferences for information delivery through the
Table 1 Overall descriptive statistics

\begin{tabular}{|c|c|c|c|}
\hline Variable & $\begin{array}{l}\text { Total Sample } \\
N=942(100 \%)\end{array}$ & $\begin{array}{l}\text { Pregnant } \\
N=358(38 \%)\end{array}$ & $\begin{array}{l}\text { Non-pregnant } \\
N=584(62 \%)\end{array}$ \\
\hline Age (mean, SD) & $31.8(4.7)$ & $30.8(5.4)$ & $32.4(4.2)^{* *}$ \\
\hline \multicolumn{4}{|l|}{ Ethnicity (n, \%) } \\
\hline Dutch & $884(93.8)$ & $332(92.7)$ & $552(94.5)$ \\
\hline Other & $58(6.2)$ & $26(7.3)$ & $32(5.5)$ \\
\hline \multicolumn{4}{|l|}{ Education (n, \%) } \\
\hline Low & $83(8.8)$ & $37(10.3)$ & $46(7.9)$ \\
\hline Middle & $228(24.2)$ & $97(27.1)$ & $131(22.4)$ \\
\hline High & $631(67.0)$ & $224(62.6)$ & $407(69.7)$ \\
\hline \multicolumn{4}{|c|}{ One or more children (n, \%) } \\
\hline Yes & $797(84.6)$ & $213(59.5)$ & $584(100)$ \\
\hline No & $145(15.4)$ & $145(40.5)$ & $\mathrm{N} / \mathrm{A}$ \\
\hline \multicolumn{4}{|c|}{ Age youngest child (n, \%) } \\
\hline $0-2$ years & $646(81.1)$ & $62(29.1)$ & $584(100)$ \\
\hline $2-4$ years & $98(12.3)$ & $98(46.0)$ & $\mathrm{N} / \mathrm{A}$ \\
\hline $4-8$ years & $35(4.4)$ & $35(16.4)$ & $\mathrm{N} / \mathrm{A}$ \\
\hline $8-12$ years & $13(1.6)$ & $13(6.1)$ & $\mathrm{N} / \mathrm{A}$ \\
\hline 12-18 years & $3(0.4)$ & $3(1.4)$ & $\mathrm{N} / \mathrm{A}$ \\
\hline $18+$ years & $2(0.3)$ & $2(0.9)$ & $\mathrm{N} / \mathrm{A}$ \\
\hline \multicolumn{4}{|c|}{ Occupation as Health care worker (n, \%) } \\
\hline Yes & $340(36.1)$ & $125(34.9)$ & $215(36.8)$ \\
\hline No & $602(63.9)$ & $233(65.1)$ & $369(63.2)$ \\
\hline \multicolumn{4}{|c|}{ Awareness of MPV (n, \%) } \\
\hline Aware & $576(61,1)$ & $248(69,3)$ & $328(56,2)^{* *}$ \\
\hline Unaware & $366(38,9)$ & $110(30,7)$ & $256(43,8)^{* *}$ \\
\hline \multicolumn{4}{|c|}{ Engagement with MPV (n, \%) } \\
\hline Unengaged & $147(15,6)$ & $49(13,7)$ & $98(16,8)$ \\
\hline Neutral & $240(25,5)$ & $83(23,2)$ & $157(26,9)$ \\
\hline Engaged & $555(58,9)$ & $226(63,1)$ & $329(56,3)$ \\
\hline \multicolumn{4}{|c|}{ Information seeking (n, \%) } \\
\hline Yes & $347(36,8)$ & $153(44,7)$ & $194(36,8)^{*}$ \\
\hline No & $522(55,4)$ & $189(55,3)$ & $333(63,2)^{*}$ \\
\hline Missing & $73(7,7)$ & $16(\mathrm{~N} / \mathrm{A})$ & $57(\mathrm{~N} / \mathrm{A})$ \\
\hline \multicolumn{4}{|l|}{ Vaccinated (n, \%) } \\
\hline Yes & $227(24,1)$ & $111(31)$ & $116(19,9)^{* *}$ \\
\hline No & $715(75,9)$ & $247(69)$ & $468(80,1)^{* *}$ \\
\hline
\end{tabular}

c N/A = not applicable. ${ }^{*}<.05 ;{ }^{* *} p<.000$

internet (35\%) or in consultation with a HCW (35\%). Pregnant women who already had a child, and nonpregnant women who were $\mathrm{HCW}$ themselves were more likely to be aware of the MPV ( 64 and $42 \%$ respectively).

Examining the women who were unaware of MPV, the majority of both pregnant and non-pregnant women reported a need for information and preferred the midwife to be the source of MPV information. For non-pregnant 
Table 2 Descriptive statistics Stage 1: Awareness of MPV (overall aware $N=576$ )

\begin{tabular}{|c|c|c|c|c|}
\hline & \multicolumn{2}{|l|}{ Pregnant $(\mathrm{N}=358)$} & \multicolumn{2}{|l|}{ Non-pregnant $(\mathrm{N}=584)$} \\
\hline & Aware $(N=248(69.3))$ & Unaware $(N=110(30.7))$ & Aware $(N=328(56.2))$ & Unaware $(N=256(43.8))$ \\
\hline Age $(M, S d)$ & $31.1(5.6)$ & $30.3(4.6)$ & $32.2(4.1)$ & $32.6(4.3)$ \\
\hline \multicolumn{5}{|l|}{ Ethnicity (N, \%) } \\
\hline Dutch & $227(91.5)$ & $105(95.5)$ & $309(94.2)$ & $243(94.9)$ \\
\hline Other & $21(8.5)$ & $5(4.5)$ & $19(5.8)$ & $13(5.1)$ \\
\hline \multicolumn{5}{|l|}{ Education (N, \%) } \\
\hline Low & $27(10.9)$ & $10(9.1)$ & $31(9.5)$ & $15(5.9)$ \\
\hline Middle & $71(28.6)$ & 26 (23.6) & $65(19.8)$ & $66(25.8)$ \\
\hline High & $150(60.5)$ & $74(67.3)$ & $232(70.7)$ & $175(68.4)$ \\
\hline \multicolumn{5}{|l|}{ Occupation as a HCW (N, \%) } \\
\hline Yes & $91(36.7)$ & $34(30.9)$ & $139(42.4)$ & $76(29.7)^{* *}$ \\
\hline No & $157(63.3)$ & $76(69.1)$ & $189(57.6)$ & $180(70.3)^{* *}$ \\
\hline \multicolumn{5}{|l|}{ One or more children $(\mathrm{N}, \%)$} \\
\hline Yes & $159(64.1)$ & $54(49.1)^{* *}$ & $328(100)$ & $256(100)$ \\
\hline No & $89(35.9)$ & $56(50.9)$ & N/A & N/A \\
\hline Information source (N, \%) & & N/A & & N/A \\
\hline Midwife & $121(48.8)$ & & 129 (39.3) & \\
\hline GP & $36(14.5)$ & & $28(8.5)$ & \\
\hline Municipal public health services & $32(12.9)$ & & $17(5.2)$ & \\
\hline Gynaecologist & $18(7.3)$ & & $28(8.5)$ & \\
\hline Youth health care worker & $22(8.9)$ & & $12(3.7)$ & \\
\hline Public Health Institute & $22(8.9)$ & & $46(14.0)$ & \\
\hline Government & $22(8.9)$ & & $23(7.0)$ & \\
\hline Pregnant women & $45(18.1)$ & & $69(21.0)$ & \\
\hline Other & $49(19.8)$ & & $91(27.7)$ & \\
\hline Information type $(\mathrm{N}, \%)$ & & N/A & & N/A \\
\hline Consultation HCW & $86(34.7)$ & & $99(30.2)$ & \\
\hline Information flyer about MPV & $75(30.2)$ & & $78(23.8)$ & \\
\hline Website of Public Health Institute & $40(16.1)$ & & $66(20.1)$ & \\
\hline Internet & $86(34.7)$ & & $114(34.8)$ & \\
\hline Social media & $27(10.9)$ & & $41(12.5)$ & \\
\hline Traditional media & $18(7.3)$ & & $45(13.7)$ & \\
\hline Centering pregnancy & $20(8.1)$ & & $31(9.5)$ & \\
\hline Other & $24(9.7)$ & & $34(10.4)$ & \\
\hline Information need (N, \%) & N/A & & N/A & \\
\hline Yes & & $94(85.5)$ & & $199(77.7)$ \\
\hline No & & $16(14.5)$ & & $57(22.3)$ \\
\hline Information source $(\mathrm{N}, \%)^{a}$ & N/A & & N/A & \\
\hline Midwife & & $65(69.1)$ & & $150(75.4)$ \\
\hline GP & & $11(11.7)$ & & $3(1.5)$ \\
\hline Municipal public health services & & $3(3.2)$ & & $3(1.5)$ \\
\hline Gynaecologist & & $9(9.6)$ & & $26(13.1)$ \\
\hline Youth health care worker & & $1(1.1)$ & & $6(3.0)$ \\
\hline Public Health Institute & & $2(2.1)$ & & $8(4.0)$ \\
\hline
\end{tabular}


Table 2 Descriptive statistics Stage 1: Awareness of MPV (overall aware $N=576)$ (Continued)

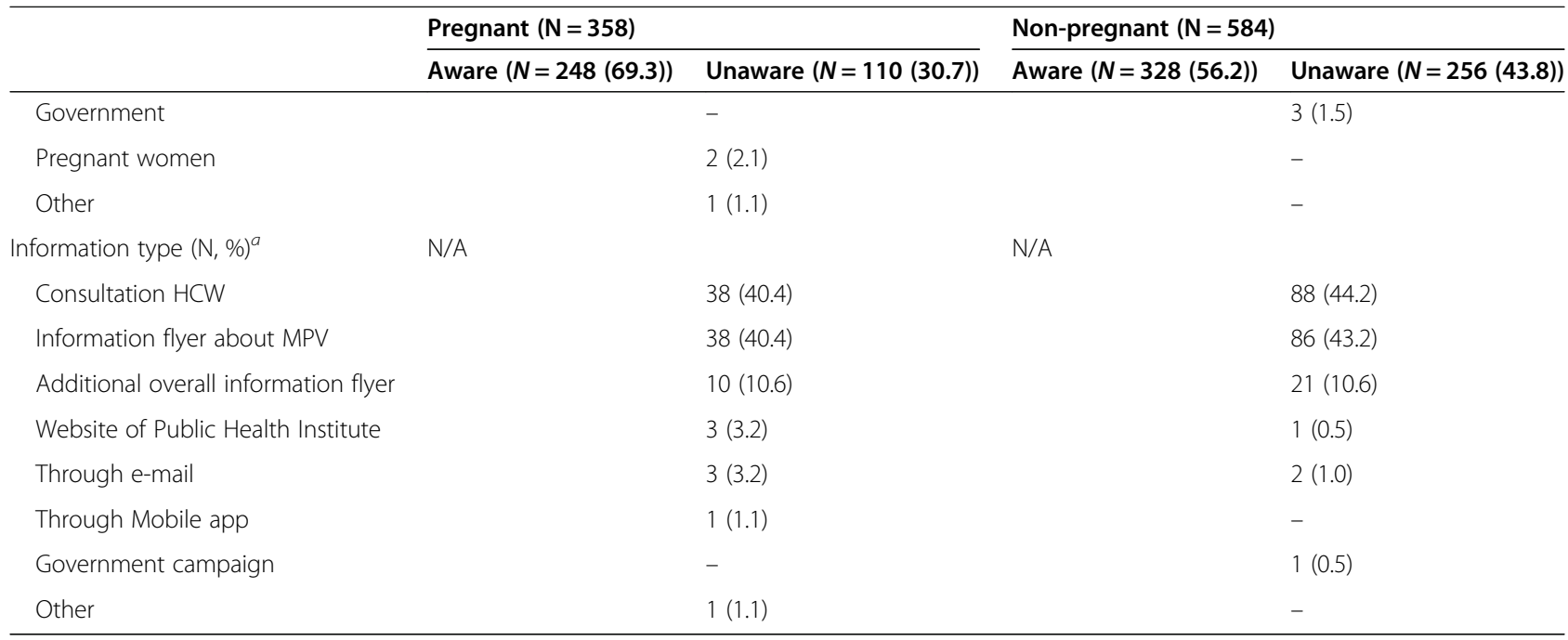

a routing only when need for information was yes. $\mathrm{M}=$ Mean; $\mathrm{Sd}=$ standard deviation; $\mathrm{N}=$ number of participants; $\%=$ percentage of participants; $\mathrm{N} / \mathrm{A}=$ not applicable. ${ }^{*} p<.05 ; * * 0.01$

women, the second most preferred information source after midwives was their gynaecologist (13\%), and for pregnant women their GP (12\%). Furthermore, both pregnant and non-pregnant preferred to receive information through a consultation with a $\mathrm{HCW}$ or to receive a flyer about the MPV. Women who reported to be unaware of the MPV, and felt no need to be informed, reported mostly that they were not interested or felt the MPV was unnecessary (respectively 37.5 and 22.8\%). Pregnant women who were not aware of the MPV also reported having received too much information regarding all aspects of pregnancy overall and felt that the MPV was not relevant to them at the current point of their pregnancy.

\section{Stage 2: engagement with MPV}

Nearly a third of both pregnant and non-pregnant women felt MPV was not important to them and were thus considered "unengaged" (Table 3 ). The women who were engaged with MPV were significantly older than the unengaged group. Engaged women also had more trust in their midwives, PHI and PHS. Furthermore, women engaged with MPV scored higher in measures of critical thinking (i.e. ability to distinguish different information about MPV and take into account the reliability of the information source) and descriptive norms (i.e. felt that most women will get vaccinated during their pregnancy) compared to women who were neutral or unengaged.

\section{Stage $2 b$ : information-seeking behaviour}

Only women categorised as neutral or engaged in Stage 2 can enter Stage 2b of information-seeking behaviour. Of the neutral and engaged women, about $60 \%$ had actively searched for information regarding MPV (Table 4). Women who had searched for information were mostly higher educated and scored higher on selfefficacy (i.e. considered themselves able to decide MPV). Information-seeking women also scored higher in measures of critical thinking, subjective knowledge (i.e. how good they thought their knowledge about MPV was), and trust in the PHI. These women were most often classified as having a rational decision-making style.

The PHI website was most often used as a source for (additional) information in both pregnant and nonpregnant women $(34.1 \%$ vs $45.9 \%$ respectively), followed by their midwife/gynaecologist (19.4\% vs $14.5 \%$ respectively). Furthermore, pregnant women reported that their GP was a source of MPV information (11.5\%), while non-pregnant women used other websites (11.7\%). These websites were mostly Google or forum-type websites. However, trust in "other websites" was lower compared to other information sources.

Non-pregnant women who had searched for information were more often of Dutch descent, and/or were in the health care profession. Non-pregnant women who did not search for information mostly had an avoidant decision-making style. Pregnant women who searched for information mostly did so during their first (38.9\%) or second (35.9\%) trimester and reported higher trust in their midwives.

\section{Stage 3: vaccination behaviour (i.e. acting)}

Only women with a neutral or engaged score in Stage 2 can enter this stage, irrespective of passing through Stage 2B. Of those, approximately $43 \%$ of pregnant women and $38 \%$ of non-pregnant women were vaccinated with the MPV during their most current or most 
Table 3 Descriptive statistics stage 2: Engagement with MPV (Overall engaged $N=500$ )

\begin{tabular}{|c|c|c|c|c|c|c|}
\hline & \multicolumn{3}{|l|}{ Pregnant $(\mathrm{N}=248)$} & \multicolumn{3}{|c|}{ Non-pregnant $(\mathrm{N}=328)$} \\
\hline & $\begin{array}{l}\text { Unengaged }(N=31 \\
(12.5))\end{array}$ & $\begin{array}{l}\text { Neutral }(\mathrm{N}=50 \\
(20.2))\end{array}$ & $\begin{array}{l}\text { Engaged }(N=167 \\
(67.3))\end{array}$ & $\begin{array}{l}\text { Unengaged }(N=45 \\
(13.7))\end{array}$ & $\begin{array}{l}\text { Neutral }(N=74 \\
(22.6))\end{array}$ & $\begin{array}{l}\text { Engaged }(N=209 \\
(63.7))\end{array}$ \\
\hline Age $(M, S d)$ & $30.1(4.2)$ & $29.5(5.4)^{\mathrm{e}}$ & $31.7(5.8)^{* n}$ & $30.7(3.4)^{\mathrm{e}}$ & $31.7(3.8)$ & $32.7(4.3)^{* * 4}$ \\
\hline \multicolumn{7}{|l|}{ Ethnicity (N, \%) } \\
\hline Dutch & $28(90.3)$ & $45(90.0)$ & $154(92.2)$ & $42(93.3)$ & 70 (94.6) & $197(94.3)$ \\
\hline Other & $3(9.7)$ & $5(10.0)$ & $13(7.8)$ & $3(6.7)$ & $4(5.4)$ & $12(5.7)$ \\
\hline \multicolumn{7}{|l|}{ Education (N, \%) } \\
\hline Low \& middle & $11(35.5)$ & $23(46)$ & $64(38.3)$ & $18(40.0)$ & $23(31.1)$ & $55(26.3)$ \\
\hline High & $20(64.5)$ & $27(54)$ & $103(61.7)$ & $27(60.0)$ & $51(68.9)$ & $154(73.7)$ \\
\hline \multicolumn{7}{|c|}{ Occupation as a HCW (N, \%) } \\
\hline Yes & $12(38.7)$ & $13(26.0)$ & $66(39.5)$ & $19(42.2)$ & $28(37.8)$ & $92(44.0)$ \\
\hline No & $19(61.3)$ & $37(74.0)$ & $101(60.5)$ & $26(57.8)$ & $46(62.2)$ & $117(56.0)$ \\
\hline \multicolumn{7}{|c|}{ One or more children $(\mathrm{N}, \%)$} \\
\hline Yes & $19(61.3)$ & $25(50.0)^{\mathrm{e}}$ & $115(68.9)^{* n}$ & $45(100)$ & $74(100)$ & $209(100)$ \\
\hline No & $12(38.7)$ & $25(50.0)^{\mathrm{e}}$ & $52(31.1)^{* n}$ & N/A & N/A & N/A \\
\hline $\begin{array}{l}\text { Critical thinking }{ }^{\mathrm{a}}(\mathrm{M}, \\
\mathrm{Sd})\end{array}$ & $4.8(1.5)^{\mathrm{e}}$ & $4.8(1.0)^{\mathrm{e}}$ & $5.5(0.9)^{* * * u . n}$ & $4.9(1.1)^{\mathrm{e}}$ & $5.1(0.9)^{\mathrm{e}}$ & $5.9(0.8)^{* * * 4 . n}$ \\
\hline $\begin{array}{l}\text { Descriptive norm } \\
(\mathrm{M}, \mathrm{Sd})\end{array}$ & $3.7(1.5)^{\mathrm{e}}$ & $4.1(1.0)^{\mathrm{e}}$ & $4.9(1.1)^{* * * 4.0}$ & $3.9(1.4)^{\mathrm{e}}$ & $4.1(1.2)^{\mathrm{e}}$ & $4.8(1.2)^{* * * 4 . n}$ \\
\hline \multicolumn{7}{|l|}{ Trust (M, Sd) } \\
\hline Midwife & $4.2(1.5)^{\text {n.e }}$ & $5.1(1.2)^{\text {u.e }}$ & $5.7(1.1)^{* * * u . n}$ & $5.4(1.2)$ & $5.4(1.1)^{\mathrm{e}}$ & $5.8(1.2)^{* n}$ \\
\hline $\begin{array}{l}\text { Public Health } \\
\text { Institute }\end{array}$ & $3.7(1.7)^{\text {n.e }}$ & $5.1(1.2)^{\text {u.e }}$ & $5.7(0.9)^{* * * u . n}$ & $5.1(1.6)^{\mathrm{e}}$ & $5.4(1.1)^{\mathrm{e}}$ & $6.0(1.1)^{* * * u . n}$ \\
\hline $\begin{array}{l}\text { Youth health care } \\
\text { centre }\end{array}$ & $3.4(1.8)^{\text {n.e }}$ & $4.8(1.1)^{\text {u.e }}$ & $5.3(1.1)^{* * * 4 . n}$ & $4.7(1.5)^{\mathrm{e}}$ & $4.9(1.2)^{e}$ & $5.4(1.3)^{* * * u . n}$ \\
\hline
\end{tabular}

$\mathrm{M}=$ Mean; $\mathrm{Sd}=$ standard deviation; $\mathrm{N}=$ number of participants; $\%=$ percentage of participants; $\mathrm{u}=$ unengaged; $\mathrm{n}=$ neutral; $\mathrm{e}=$ engaged; ${ }^{*} \mathrm{p}<.05 ;{ }^{* *} \mathrm{p}<.01 ; * * *$ $p<.001 .{ }^{a}$ scale $1-7$

recent pregnancy (Table 5). Of the currently pregnant women, only 33\% were eligible for MPV based on their reported gestational age (28-36 weeks pregnant), of whom $58 \%$ were vaccinated.

Of both pregnant and non-pregnant women, women who had searched for information and felt they knew enough to make a decision (i.e. self-efficacy) were more often vaccinated. Furthermore, both pregnant and nonpregnant vaccinated women more positive intention and attitude towards MPV felt better able to distinguish different information about MPV, expected other women to also get vaccinated during their pregnancies, and felt that loved ones and HCWs would appreciate it if they would get vaccinated during their pregnancy than nonvaccinated women. Vaccinated women also felt their knowledge about MPV was better, and compared to non-vaccinated women the following considerations were more important to the vaccinated group: "Baby needs one vaccination less"; "receive vaccination through midwife"; "know how to receive MPV".

Vaccinated pregnant women rated the consideration "Baby would need first vaccination at a later age" of higher importance, and were most frequently of avoidant and impulsive decision-making styles. Vaccinated pregnant women reported greater trust in their midwife, PHI, and PHS, and more often had an older child the unvaccinated pregnant women. Vaccinated pregnant women were more frequently middle-level educated than unvaccinated pregnant women, who were mostly highly educated. Compared to vaccinated non-pregnant women, unvaccinated non-pregnant women more often of Dutch descent.

The most frequently reported reason for not being vaccinated in both pregnant and non-pregnant women was not knowing MPV existed (35\% vs $24 \%$ respectively). For pregnant women, the next most common reasons were: "I am not yet eligible to receive MPV because my gestational age is less than 28 weeks" (21\%) and "MPV was not included in the NIP" (15\%). For non-pregnant women the next most common reasons were that they "did not know enough about MPV" (22\%), "MPV was not included in the NIP" (19\%), and that their "HCW did not advise them to get vaccinated during their earlier pregnancy" (18\%). 
Table 4 Descriptive statistics stage 2b: Information seeking behaviour (Overall $N=307$ searched for information)

\begin{tabular}{|c|c|c|c|c|}
\hline & \multicolumn{2}{|l|}{ Pregnant $(N=217)$} & \multicolumn{2}{|l|}{ Non-pregnant $(N=283)$} \\
\hline & $\begin{array}{l}\text { Sought information }(N= \\
131(60.4))\end{array}$ & $\begin{array}{l}\text { Did not seek information } \\
(N=86(39.6))\end{array}$ & $\begin{array}{l}\text { Sought information }(N= \\
176(62.2))\end{array}$ & $\begin{array}{l}\text { Did not seek information } \\
(N=107(37.8))\end{array}$ \\
\hline Age $(M, S d)$ & $31.2(5.7)$ & $31.3(6.0)$ & $32.6(4.1)$ & $32.3(4.4)$ \\
\hline \multicolumn{5}{|l|}{ Ethnicity (N, \%) } \\
\hline Dutch & $120(91.6)$ & 79 (91.9) & $171(97.2)$ & $96(89.7)^{* *}$ \\
\hline Other & $11(8.4)$ & $7(8.1)$ & $5(2.8)$ & $11(10.3)^{* *}$ \\
\hline \multicolumn{5}{|l|}{ Education (N, \%) } \\
\hline Low & $8(6.1)$ & $15(17.4)^{* *}$ & $11(6.3)$ & $17(15.9)^{*}$ \\
\hline Middle & $34(26.0)$ & $30(34.9)$ & $28(15.9)$ & $22(20.6)$ \\
\hline High & $89(67.9)$ & $41(47.7)^{* *}$ & $137(77.8)$ & $68(63.6)^{*}$ \\
\hline \multicolumn{5}{|l|}{ Occupation as a HCW (N, \%) } \\
\hline Yes & $54(41.2)$ & $25(29.1)$ & $85(48.3)$ & $35(32.7)^{*}$ \\
\hline No & $77(58.8)$ & $61(70.9)$ & $91(51.7)$ & $72(67.3)^{*}$ \\
\hline \multicolumn{5}{|l|}{ Children (N, \%) } \\
\hline Yes & $84(64.1)$ & $56(65.1)$ & $176(100)$ & $107(100)$ \\
\hline No & $47(35.9)$ & $30(34.9)$ & N/A & N/A \\
\hline Time of search $(\mathrm{N}, \%)$ & & N/A & & N/A \\
\hline Before pregnancy & $17(13.0)$ & & $6(3.4)$ & \\
\hline 1st trimester & $51(38.9)$ & & $31(17.6)$ & \\
\hline 2nd trimester & $47(35.9)$ & & $85(48.3)$ & \\
\hline 3rd trimester & $13(9.9)$ & & $48(27.3)$ & \\
\hline After pregnancy & $3(2.3)$ & & $6(3.4)$ & \\
\hline \multicolumn{5}{|l|}{ Decision-making style $(\mathrm{M}, \mathrm{Sd})$} \\
\hline Rational & $4.0(0.5)$ & $3.8(0.6)^{* *}$ & $4.1(0.5)$ & $3.9(0.5)^{* *}$ \\
\hline Intuitive & $3.6(0.7)$ & $3.6(0.7)$ & $3.5(0.7)$ & $3.7(0.7)$ \\
\hline Dependent & $3.6(0.6)$ & $3.6(0.7)$ & $3.4(0.7)$ & $3.4(0.6)$ \\
\hline Avoidant & $2.8(1.0)$ & $2.8(1.0)$ & $2.2(0.9)$ & $2.5(0.9)^{*}$ \\
\hline Impulsive & $3.0(0.8)$ & $3.1(0.7)$ & $2.8(0.7)$ & $2.9(0.7)$ \\
\hline Critical Thinking ${ }^{a}(M, S d)$ & $5.6(0.8)$ & $4.9(1.1)^{* * *}$ & $5.9(0.8)$ & $5.4(0.9)^{* * *}$ \\
\hline Descriptive norm ${ }^{\mathrm{a}}(\mathrm{M}, \mathrm{Sd})$ & $4.8(1.1)$ & $4.6(1.2)$ & $4.7(1.2)$ & $4.6(1.2)$ \\
\hline \multicolumn{5}{|l|}{ Injunctive norm ${ }^{\mathrm{a}}(\mathrm{M}, \mathrm{Sd})$} \\
\hline Loved ones & $4.8(1.3)$ & $4.7(1.4)$ & $5.0(1.2)$ & $4.8(1.2)$ \\
\hline Health care worker & $4.8(1.4)$ & $4.7(1.4)$ & $5.0(1.3)$ & $4.8(1.2)$ \\
\hline \multicolumn{5}{|l|}{ Trust $(\mathrm{M}, \mathrm{Sd})^{\mathrm{a}}$} \\
\hline Midwife & $5.7(1.1)$ & $5.3(1.2)^{*}$ & $5.7(1.3)$ & $5.6(1.1)$ \\
\hline Public Health Institute & $5.7(1.0)$ & $5.2(1.0)^{* * *}$ & $6.0(1.1)$ & $5.6(1.1)^{* *}$ \\
\hline $\begin{array}{l}\text { Youth Health Care } \\
\text { centre }\end{array}$ & $5.3(1.1)$ & $5.1(1.1)$ & $5.3(1.4)$ & $5.3(1.2)$ \\
\hline $\begin{array}{l}\text { Subjective Knowledge }(M, \\
\text { Sd) }\end{array}$ & $3.7(0.7)$ & $3.1(1.0)^{* * *}$ & $3.8(0.9)$ & $3.0(1.0)^{* * *}$ \\
\hline \multicolumn{5}{|l|}{ Self-efficacy (N, \%) } \\
\hline Yes & $113(86.3)$ & $50(58.1)^{* * *}$ & $142(80.7)$ & $65(60.7)^{* * *}$ \\
\hline No & $18(13.7)$ & $36(41.9)^{* * *}$ & $34(19.3)$ & $42(39.3)^{* * *}$ \\
\hline Information source (N, \%) & & N/A & & N/A \\
\hline GP & 25 (11.5) & & $22(7.8)$ & \\
\hline
\end{tabular}


Table 4 Descriptive statistics stage 2b: Information seeking behaviour (Overall $N=307$ searched for information) (Continued)

\begin{tabular}{|c|c|c|c|c|}
\hline & \multicolumn{2}{|l|}{ Pregnant $(N=217)$} & \multicolumn{2}{|l|}{ Non-pregnant $(N=283)$} \\
\hline & $\begin{array}{l}\text { Sought information }(N= \\
131(60.4))\end{array}$ & $\begin{array}{l}\text { Did not seek information } \\
(N=86(39.6))\end{array}$ & $\begin{array}{l}\text { Sought information }(N= \\
176(62.2))\end{array}$ & $\begin{array}{l}\text { Did not seek information } \\
(N=107(37.8))\end{array}$ \\
\hline Midwife/Gynaecologist & $42(19.4)$ & & $41(14.5)$ & \\
\hline $\begin{array}{l}\text { Youth health care } \\
\text { worker }\end{array}$ & $12(5.5)$ & & $9(3.2)$ & \\
\hline $\begin{array}{l}\text { Municipal public health } \\
\text { services }\end{array}$ & $19(8.8)$ & & $22(7.8)$ & \\
\hline $\begin{array}{l}\text { Website of Public Health } \\
\text { Institute }\end{array}$ & $74(34.1)$ & & $130(45.9)$ & \\
\hline Other Website & $15(6.9)$ & & $33(11.7)$ & \\
\hline Scientific articles & $11(5.1)$ & & $16(5.7)$ & \\
\hline Television & $2(0.9)$ & & $3(1.1)$ & \\
\hline Newspaper/magazine & $2(0.9)$ & & $1(0.4)$ & \\
\hline Pregnant women & $18(8.3)$ & & $18(6.4)$ & \\
\hline Social media & $9(4.1)$ & & $14(4.9)$ & \\
\hline Family/friends & $10(4.6)$ & & $6(2.1)$ & \\
\hline Other & $4(1.8)$ & & $2(0.7)$ & \\
\hline Information Trust ${ }^{\mathrm{a}}(\mathrm{M}, \mathrm{Sd})$ & & $\mathrm{N} / \mathrm{A}$ & & N/A \\
\hline GP & $5.8(1.0)$ & & $5.1(1.8)$ & \\
\hline Midwife/Gynaecologist & $5.8(1.1)$ & & $5.7(1.3)$ & \\
\hline $\begin{array}{l}\text { Youth health care } \\
\text { worker }\end{array}$ & $6.0(0.9)$ & & $5.4(1.3)$ & \\
\hline $\begin{array}{l}\text { Municipal public health } \\
\text { services }\end{array}$ & $5.7(1.0)$ & & $6.1(0.9)$ & \\
\hline $\begin{array}{l}\text { Website of Public Health } \\
\text { Institute }\end{array}$ & $6.0(0.8)$ & & $5.9(1.4)$ & \\
\hline Other Website & $4.4(0.5)$ & & $4.6(1.3)$ & \\
\hline Scientific articles & $4.9(1.8)$ & & $5.9(1.3)$ & \\
\hline Television & $5.0(0.0)$ & & $5.0(1.0)$ & \\
\hline Newspaper/magazine & $5.0(1.4)$ & & $3.0(\mathrm{~N} / \mathrm{A})$ & \\
\hline Pregnant women & $4.5(0.9)$ & & $4.1(1.5)$ & \\
\hline Social media & $3.6(0.7)$ & & $3.7(1.5)$ & \\
\hline Family/friends & $5.9(1.1)$ & & $5.8(0.8)$ & \\
\hline Other & $5.8(1.9)$ & & $4.5(0.7)$ & \\
\hline
\end{tabular}

\section{Discussion}

\section{Main results}

Before the inclusion of the MPV in the Dutch NIP at the end of 2019, when MPV was only available as an out-ofpocket purchase, almost two-thirds of women were aware of the MPV. Awareness was higher among women who were pregnant at the time of survey completion compared to women who were not pregnant but had a child younger than two years (respectively 69\% versus 56\%). Awareness among pregnant women was likely higher due to being actively informed about the MPV by their midwife. Vaccine uptake was 58\% among engaged pregnant women who were eligible for vaccination, and
$38 \%$ among engaged non-pregnant women. Furthermore, despite different vaccination rates between groups, most women were classified as engaged as they felt that MPV was an important topic to them. As non-pregnant women were less likely to have encountered the new MPV communication materials issued in 2017 and 2018, the lower awareness and vaccine uptake of MPV in this group indicates that the additional communication efforts to improve awareness of MPV seem to have been a success.

The information leaflets, flyers, and posters used for MPV information communication are easily implemented in daily practice. As such, these methods could 
Table 5 Descriptive statistics stage 3: Vaccine uptake (Overall $N=199$ were vaccinated)

\begin{tabular}{|c|c|c|c|c|}
\hline & \multicolumn{2}{|l|}{ Pregnant $(N=217)$} & \multicolumn{2}{|c|}{ Non-pregnant $(\mathrm{N}=\mathbf{2 8 3})$} \\
\hline & $\begin{array}{l}\text { Vaccinated }(N=93 \\
(42.9))\end{array}$ & $\begin{array}{l}\text { Not vaccinated }(N=124 \\
(57.1))\end{array}$ & $\begin{array}{l}\text { Vaccinated }(N=106 \\
(37.5))\end{array}$ & $\begin{array}{l}\text { Not vaccinated }(N=177 \\
(62.5))\end{array}$ \\
\hline Age $(M, S d)$ & $31.7(6.2)$ & $30.9(5.5)$ & $32.6(4.7)$ & $32.4(3.8)$ \\
\hline \multicolumn{5}{|l|}{ Ethnicity (N, \%) } \\
\hline Dutch & $82(88.2)$ & $117(94.4)$ & $96(90.6)$ & $171(96.6)^{*}$ \\
\hline Other & $11(11.8)$ & $7(5.6)$ & $10(9.4)$ & $6(3.4)^{*}$ \\
\hline \multicolumn{5}{|l|}{ Education (N, \%) } \\
\hline Low & $12(12.9)$ & $11(8.9)$ & $7(6.6)$ & $21(11.9)$ \\
\hline Middle & $37(39.8)$ & $27(21.8)^{* *}$ & $15(14.2)$ & 35 (19.8) \\
\hline High & $44(47.3)$ & $86(69.4)^{* *}$ & $84(79.2)$ & $121(68.4)$ \\
\hline \multicolumn{5}{|l|}{ Occupation as a HCW (N, \%) } \\
\hline Yes & $36(38.7)$ & $43(34.7)$ & $48(45.3)$ & $72(40.7)$ \\
\hline No & $57(61.3)$ & $81(65.3)$ & $58(54.7)$ & $105(59.3)$ \\
\hline \multicolumn{5}{|l|}{ Children (N, \%) } \\
\hline Yes & $67(72.0)$ & $73(58.9)^{*}$ & $106(100)$ & $177(100)$ \\
\hline No & $26(28.0)$ & $51(41.1)^{*}$ & N/A & N/A \\
\hline \multicolumn{5}{|l|}{ Age youngest child (N, \%) } \\
\hline $0-2$ yrs. & $16(23.9)$ & $22(30.1)$ & $106(100)$ & $177(100)$ \\
\hline $2-4 \mathrm{yrs}$ & $28(41.8)$ & $38(52.1)$ & N/A & N/A \\
\hline $4-8 \mathrm{yrs}$. & $11(16.4)$ & $11(15.1)$ & N/A & N/A \\
\hline $8-12$ yrs. & $10(14.9)$ & $2(2.7)$ & N/A & N/A \\
\hline $12-18 \mathrm{yrs}$ & $2(3.0)$ & $0(0)$ & N/A & N/A \\
\hline $18 \mathrm{yrs}$. and older & $0(0)$ & $0(0)$ & N/A & N/A \\
\hline \multicolumn{5}{|l|}{ Considerations $^{\mathrm{a}}(\mathrm{M}, \mathrm{Sd})$} \\
\hline 1) Baby 1 less vaccination & $5.5(1.2)$ & $5.1(1.4)^{*}$ & $5.6(1.6)$ & $5.1(1.6)^{*}$ \\
\hline 2) Receive vaccine midwife & $5.4(1.1)$ & $4.9(1.6)^{* *}$ & $5.6(1.4)$ & $5.1(1.6)^{* *}$ \\
\hline 3) Know how to get MPV & $5.6(1.1)$ & $4.9(1.5)^{* * *}$ & $6.1(1.1)$ & $4.9(1.6)^{* * *}$ \\
\hline 4) MPV included in NIP & $5.5(1.3)$ & $5.3(1.6)$ & $5.5(1.5)$ & $5.3(1.5)$ \\
\hline 5) Baby vaccinated later age & $5.4(1.1)$ & $5.0(1.6)^{*}$ & $5.4(1.7)$ & $5.1(1.7)$ \\
\hline $\begin{array}{l}\text { 6) MPV not through youth health } \\
\text { care centre }\end{array}$ & $5.5(1.3)$ & $5.3(1.2)$ & $5.9(1.2)$ & $5.6(1.2)$ \\
\hline \multicolumn{5}{|l|}{ Decision-making style $(\mathrm{M}, \mathrm{Sd})$} \\
\hline Rational & $4.0(0.5)$ & $3.9(0.5)$ & $4.1(0.5)$ & $4.0(0.5)$ \\
\hline Intuitive & $3.7(0.7)$ & $3.6(0.7)$ & $3.6(0.7)$ & $3.6(0.7)$ \\
\hline Dependent & $3.7(0.6)$ & $3.6(0.7)$ & $3.5(0.7)$ & $3.4(0.6)$ \\
\hline Avoidant & $3.0(1.0)$ & $2.6(0.9)^{* *}$ & $2.3(1.0)$ & $2.4(0.9)$ \\
\hline Impulsive & $3.2(0.8)$ & $2.9(0.7)^{* *}$ & $2.9(0.7)$ & $2.8(0.7)$ \\
\hline Critical Thinking $^{\mathrm{a}}(\mathrm{M}, \mathrm{Sd})$ & $5.5(0.9)$ & $5.2(1.0)^{*}$ & $5.9(0.9)$ & $5.6(0.9)^{* *}$ \\
\hline Descriptive norm ${ }^{a}(\mathrm{M}, \mathrm{Sd})$ & $5.1(1.0)$ & $4.5(1.1)^{* * *}$ & $5.0(1.1)$ & $4.5(1.2)^{* * *}$ \\
\hline \multicolumn{5}{|l|}{ Injunctive norm ${ }^{\mathrm{a}}(\mathrm{M}, \mathrm{Sd})$} \\
\hline Loved ones & $5.2(1.1)$ & $4.4(1.3)^{* * *}$ & $5.4(1.1)$ & $4.7(1.2)^{* * *}$ \\
\hline $\mathrm{HCW}$ & $5.2(1.3)$ & $4.5(1.4)^{* *}$ & $5.3(1.3)$ & $4.7(1.3)^{* * *}$ \\
\hline \multicolumn{5}{|l|}{ Trust $^{a}(\mathrm{M}, \mathrm{Sd})$} \\
\hline Midwife & $5.8(1.0)$ & $5.4(1.2)^{* *}$ & $5.7(1.3)$ & $5.7(1.1)$ \\
\hline Public Health Institute & $5.7(1.0)$ & $5.4(1.1)^{*}$ & $6.0(1.1)$ & $5.8(1.1)$ \\
\hline
\end{tabular}


Table 5 Descriptive statistics stage 3: Vaccine uptake (Overall $N=199$ were vaccinated) (Continued)

\begin{tabular}{|c|c|c|c|c|}
\hline & \multicolumn{2}{|l|}{ Pregnant $(\mathrm{N}=217)$} & \multicolumn{2}{|c|}{ Non-pregnant $(\mathrm{N}=283)$} \\
\hline & $\begin{array}{l}\text { Vaccinated }(N=93 \\
(42.9))\end{array}$ & $\begin{array}{l}\text { Not vaccinated }(N=124 \\
(57.1))\end{array}$ & $\begin{array}{l}\text { Vaccinated }(N=106 \\
(37.5))\end{array}$ & $\begin{array}{l}\text { Not vaccinated }(N=177 \\
(62.5))\end{array}$ \\
\hline Youth Health Care centre & $5.4(0.9)$ & $5.0(1.2)^{* *}$ & $5.3(1.4)$ & $5.3(1.3)$ \\
\hline Subjective Knowledge ${ }^{a}(\mathrm{M}, \mathrm{Sd})$ & $3.9(0.6)$ & $3.2(0.9)^{* * *}$ & $4.0(0.8)$ & $3.2(1.0)^{* * *}$ \\
\hline Intention ${ }^{\mathrm{a}}(\mathrm{M}, \mathrm{Sd})$ & $5.8(1.1)$ & $4.8(1.5)^{* * *}$ & $6.3(1.1)$ & $4.9(1.6)^{* * *}$ \\
\hline Attitude $^{a}(\mathrm{M}, \mathrm{Sd})$ & $5.4(1.0)$ & $4.8(1.1)^{* * *}$ & $5.9(1.0)$ & $5.0(1.3)^{* * *}$ \\
\hline \multicolumn{5}{|l|}{ Self-efficacy (N, \%) } \\
\hline Yes & $83(89.2)$ & $80(64.5)^{* * *}$ & $101(95.3)$ & $106(59.9)^{* * *}$ \\
\hline No & $10(10.8)$ & $44(35.5)^{* * *}$ & $5(4.7)$ & $71(40.1)^{* * *}$ \\
\hline \multicolumn{5}{|l|}{ Information seeking $(\mathrm{N}, \%)$} \\
\hline Yes & $68(73.1)$ & $63(50.8)^{* *}$ & $78(73.6)$ & $98(55.4)^{* *}$ \\
\hline No & $25(26.9)$ & $61(49.2)^{* *}$ & $28(26.4)$ & $79(44.6)^{* *}$ \\
\hline Vaccine provider $(\mathrm{N}, \%)$ & & N/A & & N/A \\
\hline GP & $48(51.6)$ & & $44(41.5)$ & \\
\hline Midwife & 19 (20.4) & & $17(16.0)$ & \\
\hline Municipal public health services & $22(23.7)$ & & $35(33.0)$ & \\
\hline Other & $4(4.3)$ & & $10(9.4)$ & \\
\hline Reason for not vaccinating (N, \%) & N/A & & N/A & \\
\hline Did not know MPV existed & & $36(29.0)$ & & $34(19.2)$ \\
\hline Objections against MPV & & $9(7.3)$ & & $12(6.8)$ \\
\hline Did not know enough about MPV & & $9(7.3)$ & & $37(20.9)$ \\
\hline Couldn't afford MPV & & $4(3.2)$ & & $5(2.8)$ \\
\hline MPV is not included in NIP & & $19(15.3)$ & & $34(19.2)$ \\
\hline MPV was not advised by HCW & & $11(8.9)$ & & $29(16.4)$ \\
\hline Other & & $36(29.0)$ & & $26(14.7)$ \\
\hline
\end{tabular}

also be considered for other non-NIP vaccines, such as the Rotavirus vaccine and the human papillomavirus (HPV) vaccine for boys. While providing information regarding additional, non-NIP vaccines is not standard practice in many countries, this may be a feasible method that seems to increase awareness of additional vaccines among the public, as well as among HCWs.

\section{Awareness of MPV}

The significant, overall difference in awareness between pregnant and non-pregnant women could be explained by information provision routes relying on midwives. This information route (through consultation and information flyers) was more accessible to, and more frequently reported by, pregnant women than nonpregnant women. Of non-pregnant women who were aware of MPV, most reported having used the PHI website and traditional media as an information source. These sources were also available to (and used by) pregnant women. Results from previous studies have shown that overall awareness of a topic can be increased by using multiple information delivery routes [15-17]. In this study, most women who were unaware of the MPV indicated needing further information and preferred to be informed by their midwives, either through in-person consultation or by receiving a flyer about MPV. Providing information regarding additional vaccines is not a standard procedure in many countries. However, it is feasible and seems to increase awareness about additional vaccines among public as well as HCWs.

\section{Engagement with MPV}

Most aware women indicated MPV was important to them. These women considered themselves better able to distinguish information and assess the reliability of particular information and its source, and felt that most women would get vaccinated during their pregnancy. These results can be explained by certain cognitive biases; mental short-cuts people make when making decisions. For example, people tend to think that their beliefs and actions are relatively widespread through the general population; known as consensus bias. People 
also attribute positive terms, such as the ability to distinguish information and assess the reliability of information sources, more often to themselves than others; known as blind-spot bias [18-21].. This might especially be relevant to topics people consider important and they will tend to think other people agree with them [22, 23].

Pregnant women who were aware of, but unengaged with MPV scored lower on trust in all three information sources (e.g. PHI, midwives, and PHS), whilst unengaged non-pregnant women who were aware of the MPV scored lower on trust in PHI and PHS. Other research has shown that trust in information sources is as essential as the actual content of the information $[24,25]$.

\section{Information seeking behaviour}

Although all women in this stage were engaged, pregnant women may have considered the MPV to be more relevant to them, as most of them still had to decide about MPV soon. This increased situational topic salience for pregnant women may have increased the likelihood of searching for information to support their imminent MPV decision, which was seen in this study. Importantly, even women who reported to be unaware of the MPV indicated that they felt a need for information regarding the MPV. Non-pregnant women mostly reported having used the PHI website to search for information. This underscores that the extra communication efforts information materials (e.g. MPV flyers provided by midwives) may have been less available to nonpregnant women at the time of their previous and most recent pregnancy. The rational decision style was most often reported among women seeking information, which is in line with our expectations and previous research [12]. A rational decision-making style requires processing a lot of information from different sources, contemplating the pros and cons of accepting MPV. This must be done whilst balancing considerations of their beliefs, experiences, and feelings about vaccination, as well as the influence of the social environment [26-28]. However, non-pregnant women who did not seek MPV information identified more as having an avoidant decision-making style. This indicates that irrespective of extra communication efforts, these women may be less likely to seek or require additional MPV information overall.

\section{Vaccination behaviour}

The abundance of available information about MPV for pregnant sample members may have had a positive influence on awareness and vaccine uptake. Relatively more engaged pregnant women reported themselves to have been vaccinated and awareness was higher compared to engaged non-pregnant women. However, other factors, such as educational level, may have played a role. Our sample consisted of relatively few higher-educated pregnant women, and higher levels of education have been previously reported to correlate with an increased hesitancy about vaccination [29-31].

Similar to engaged women, vaccinated women felt that they were more capable of distinguishing information and assessing the reliability of particular sources of information, and felt that most women will get vaccinated during their pregnancy. Among engaged pregnant women, those that were vaccinated reported higher levels of trust in all information sources, and more often already had an older child, when compared to nonvaccinated pregnant women. Having an older child may indicate women were more familiar with childhood vaccinations. As such, pregnant women with older children may have felt less overwhelmed by the influx of information about all aspects of pregnancy they received during their current pregnancy. Vaccinated engaged women also frequently stated that vaccination is "something you just do" and the decision was made without thorough deliberation, corresponding to the predominantly avoidant and impulsive decision-making styles we saw in this group. Comparable results were found in a study on vaccine acceptance among parents in the Netherlands [32]. The reason most reported for not being vaccinated during their (recent) pregnancy was engaged women did not know (enough) about MPV or not (yet) being eligible to receive MPV.

\section{Practical implications}

There are multiple 'routes' to increase awareness of vaccinations, such as websites, information flyers, and directly through HCWs. This study emphasizes that it is essential to include and reach all concerned HCWs, as they are a main, and preferred, source of information for the public. In the Netherlands, there is no direct way for the PHI to contact pregnant women. As such, we propose that the best route to further increase awareness of MPV in the Netherlands is through midwives and gynaecologists. They have an important role in providing relevant information to this target group and, according to our results, are the most trusted sources of information. Before they can begin to disseminate this knowledge to pregnant women, HCWs must firstly be engaged and educated on relevant topics, for example through e-learning [15-17]. This route to informing HCWs, and thereby the public, is relatively easily implemented and may also be effective in increasing vaccine uptake. Communication efforts about additional vaccines should therefore include routes to engage and educate HCWs.

It is may also be important to consider the timing of information provided to pregnant women about pregnancy salient topics. Women reported in this survey a feeling of being overwhelmed by the magnitude of information they received during their pregnancy. Various 
studies have demonstrated that HCWs are most capable of assessing the appropriate moments to offer information to pregnant women [15, 33-36]. However, as most pregnant women in this study searched for information about MPV during their first or second trimester, we suggest that MPV information may well be best presented no later than the beginning of the second trimester.

\section{Strengths and limitations}

To our knowledge, no previous studies have focused on increasing awareness regarding additional, non-NIP vaccines among target groups. The purpose of this study was to investigate the influence of actively informing the public about MPV through their HCWs on MPV awareness and vaccine uptake. We aimed to explore the different stages of the adapted PAPM relating to the decisionmaking process of pregnant women regarding MPV. Although this study relates to a specific vaccine and its target group, we feel our findings provide information to support future information campaigns of non-NIP vaccines, to maximise not only the spread of awareness and vaccine uptake but also public health.

However, our findings should be interpreted while considering the limitations and biases of this study. Firstly, due to the explorative cross-sectional design of this study, we cannot identify causal relationships. Further, by including non-pregnant women in our study sample, we may have introduced considerable recall biases. Questions in the survey related to the non-pregnant women's most recent pregnancy, which may have been up to a maximum of 2 years before the study. Our panel and snowballing recruitment method may also have introduced considerable selection biases. This is evidenced by the relatively high vaccine uptake in our sample (58\% of eligible pregnant women and $38 \%$ of non-pregnant women) compared to the estimated vaccination coverage in the Netherlands (26\% until April 2019) [37]. Additionally, there was no available baseline measurement of MPV awareness before the communication efforts took place, and as such, it is not possible to determine the true impact of these efforts on awareness. However, the focus of this study was to describe the (process of) PAPM during pregnancy in light of the availability of information materials. Finally, some women in Stage 3 of the survey reported that they did not know MPV existed. This result seems contradictory, as only women who indicated they were aware of MPV in earlier stages of the questionnaire could enter Stage 3. Perhaps the framing of the questions reminded women of the DTaP vaccine during the survey, indicating our question wording may require further validation.

\section{Conclusion}

As the preferred and most trusted source of information, midwives have an essential role in increasing awareness of MPV. The PHI website is an often-used source of information, but mostly after women had already become aware of MPV and felt a need for more information. Our findings demonstrate that to reach and inform the broadest range of pregnant women, best increase their awareness, and support their decision-making regarding MPV, multiple information routes are essential. Increasing awareness is the first step to improving public engagement with, and eventual acceptance of, a relatively new vaccine. To increase awareness, appropriate healthcare workers should be encouraged to actively inform target groups about available, additional vaccinations.

\section{Abbreviations}

dTaP: Diphtheria, tetanus, and acellular Pertussis vaccine; GP: General Practitioner; HCW: Health Care worker; MPV: Maternal Pertussis vaccination; NIP: National Immunisation Program; PaPM: Precaution Adoption Process Model; PHI: Public Health Institute; PHS: Public Health Services; YHW: Youth Health care Worker

\section{Supplementary Information}

The online version contains supplementary material available at https://doi. org/10.1186/s12889-021-11344-0

Additional file 1 Appendix Table 1. Cronbach's alpha.

Additional file 2.

\section{Acknowledgments}

The paper was edited by Abigail Sunderland and Fig. 1 was developed by Josta van Zoonen and the author Kim van Zoonen.

Authors' contributions

KvZ, WR and LM developed and piloted the online survey before it was sent to the external company to be programmed. KvZ analyzed and interpreted the data and wrote the main manuscript text. LM checked analyses and was a major contributor in writing the manuscript text. KvZ, WR, HdM, MB, and $\mathrm{LM}$ all read, reviewed, and approved the final manuscript.

\section{Authors' information}

Not applicable.

\section{Funding}

Not applicable.

Availability of data and materials

The datasets used and/or analyzed during the current study are available from the corresponding author on reasonable request.

\section{Declarations}

Ethics approval and consent to participate

The current study was not subject to the law in the Netherlands for medical research involving human subjects according to the ethics committee of the RIVM (The Clinical Expertise Centre RIVM; No. LCl-423). Therefore, it was exempt from needing further approval from an ethics research committee. All participants gave informed consent for their participation. All methods were carried out in accordance with relevant guidelines and regulations.

Consent for publication

Not applicable. 


\section{Competing interests}

The authors declare that they have no competing interests.

Received: 5 January 2021 Accepted: 16 June 2021

Published online: 29 June 2021

\section{References}

1. Organisation WH. WHO vaccine-preventable diseases: monitoring system. 2020 global summary: World Health Organisation; 2020. [Available from: https://apps.who.int/immunization_monitoring/globalsummary/ countries?countrycriteria\%5Bcountry\%5D\%5B\%5D=MLI\&commit=OK

2. Gezondheidsraad. Dossier: Het individuele, collectieve en publieke belang van vaccinatie. In: Gezondheidsraad, editor. Den Haag, 2013.

3. Weinstein ND, Sandman PM. The precaution adoption process model and its application. San Francisco: Jossey-Bass; 2002. p. 16-39.

4. Weinstein ND. The precaution adoption process. Health Psychol. 1988;7(4): 355-86. https://doi.org/10.1037/0278-6133.7.4.355.

5. Albery IP, Munafò M. SOCIAL COGNITIVE MODELS. Key concepts in Health Psychology. London: SAGE Publications Ltd; 2008. p. 67-71.

6. Barnard M, George P, Perryman ML, Wolff LA. Human papillomavirus (HPV) vaccine knowledge, attitudes, and uptake in college students: implications from the precaution adoption process model. PLoS One. 2017;12(8): e0182266. https://doi.org/10.1371/journal.pone.0182266.

7. Sutton S. Stages: National cancer institute - Division of cancer control \& population sciences; Available from: https://cancercontrol.cancer.gov/brp/ research/constructs/stages.html.

8. Gezondheidsraad. Vaccinatie tegen kinkhoest: doel en strategie. In: Gezondheidsraad, editor. Den Haag: Gezondheidsraad; 2015.

9. Curşeu PL, Schruijer SG. Decision styles and rationality: an analysis of the predictive validity of the general decision-making style inventory. Educational Psychological Measurement. 2012;72(6):1053-62. https://doi. org/10.1177/0013164412448066.

10. Omer SB, Betsch C, Leask J. Mandate vaccination with care: Nature Publishing Group; 2019.

11. Scott SG, Bruce R. Decision-making style: the development and assessment of a new measure. Educational psychological measurement. 1995;55(5):81831. https://doi.org/10.1177/0013164495055005017.

12. Douma L, Uiters $E$, Timmermans $D$. Decision-making styles in the context of colorectal cancer screening; 2019

13. Thunholm P. Decision-making style: habit, style, or both? Personal Individ Differ. 2004;36(4):931-44. https://doi.org/10.1016/S0191-8869(03)00162-4.

14. Netherlands S. Fertile age The Hague: Statistics Netherlands; 2020 [Available from: https://www.cbs.nl/nl-nl/onze-diensten/methoden/begrippen?tab= v\#id=vruchtbare-leeftijd.

15. Bisset KA, Paterson P. Strategies for increasing uptake of vaccination in pregnancy in high-income countries: a systematic review. Vaccine. 2018; 36(20):2751-9. https://doi.org/10.1016/.vaccine.2018.04.013.

16. Yaqub O, Castle-Clarke S, Sevdalis N, Chataway J. Attitudes to vaccination: a critical review. Soc Sci Med. 2014;112:1-11. https://doi.org/10.1016/j. socscimed.2014.04.018.

17. Puchalski S. Effect of an educational intervention on the knowledge, attitudes, and practices of pregnant women toward influenza immunization. SF Nurs Health J. 2018;2(2):2.

18. Marks $\mathrm{G}$, Miller $\mathrm{N}$. Ten years of research on the false-consensus effect: an empirical and theoretical review. Psychol Bull. 1987;102(1):72-90. https://doi. org/10.1037/0033-2909.102.1.72

19. Mullen B, Atkins JL, Champion DS, Edwards C, Hardy D, Story JE, et al. The false consensus effect: a meta-analysis of 115 hypothesis tests. J Exp Soc Psychol. 1985;21(3):262-83. https://doi.org/10.1016/0022-1031(85)90020-4.

20. Pronin $E$, Lin DY, Ross $L$. The bias blind spot: perceptions of bias in self versus others. Personal Soc Psychol Bull. 2002;28(3):369-81. https://doi.org/1 $0.1177 / 0146167202286008$

21. Scopelliti I, Morewedge CK, McCormick E, Min HL, Lebrecht S, Kassam KS. Bias blind spot: structure, measurement, and consequences. Manag Sci. 2015;61(10):2468-86. https://doi.org/10.1287/mnsc.2014.2096.

22. Latella LE, McAuley RJ, Rabinowitz M. Beliefs about vaccinations: comparing a sample from a medical school to that from the general population. Int J Environ Res Public Health. 2018;15(4):620. https://doi.org/10.3390/ijerph1504 0620
23. Rabinowitz M, Latella L, Stern C, Jost JT. Beliefs about childhood vaccination in the United States: Political ideology, false consensus, and the illusion of uniqueness. PloS one. 2016;11(7).

24. Benin AL, Wisler-Scher DJ, Colson E, Shapiro ED, Holmboe ES. Qualitative analysis of mothers' decision-making about vaccines for infants: the importance of trust. Pediatrics. 2006;117(5):1532-41. https://doi.org/10.1542/ peds.2005-1728.

25. Larson HJ. Vaccine trust and the limits of information. Science. 2016; 353(6305):1207-8. https://doi.org/10.1126/science.aah6190.

26. Sturm LA, Mays RM, Zimet GD. Parental beliefs and decision making about child and adolescent immunization: from polio to sexually transmitted infections. Journal of Developmental \& Behavioral Pediatrics. 2005;26(6):44152. https://doi.org/10.1097/00004703-200512000-00009.

27. Donaldson B, Jain P, Holder BS, Lindsey B, Regan L, Kampmann B. What determines uptake of pertussis vaccine in pregnancy? A cross-sectional survey in an ethnically diverse population of pregnant women in London. Vaccine. 2015;33(43):5822-8. https://doi.org/10.1016/.vaccine.2015.08.093.

28. Lagan BM, Sinclair M, George KW. Internet use in pregnancy informs women's decision making: a web-based survey. Birth. 2010;37(2):106-15. https://doi.org/10.1111/j.1523-536X.2010.00390.x

29. Hak E, Schönbeck Y, De Melker H, Van Essen GA, Sanders EA. Negative attitude of highly educated parents and health care workers towards future vaccinations in the Dutch childhood vaccination program. Vaccine. 2005; 23(24):3103-7. https://doi.org/10.1016/.jvaccine.2005.01.074.

30. Biasio LR. Vaccine hesitancy and health literacy. Human vaccines \& immunotherapeutics. 2017:13(3):701-2. https://doi.org/10.1080/21645515.2 016.1243633.

31. Veldwijk J, van der Heide I, Rademakers J, Schuit AJ, de Wit GA, Uiters E, et al. Preferences for vaccination: does health literacy make a difference? Med Decis Mak. 2015;35(8):948-58. https://doi.org/10.1177/0272989X1 5597225.

32. Romijnders KAGJ, Pennings J, van Osch L, de Vries H, Mollema L. Understanding how parents make decisions about childhood vaccinations [in preparation]; 2019.

33. Calo WA, Gilkey MB, Shah P, Marciniak MW, Brewer NT. Parents' willingness to get human papillomavirus vaccination for their adolescent children at a pharmacy. Prev Med. 2017;99:251-6. https://doi.org/10.1016/j.ypmed.2017. 02.003.

34. Price $L A$, Lock $L J$, Archer $L E$, Ahmed Z. Awareness of gestational diabetes and its risk factors among pregnant women in Samoa. Hawai'i Journal of Medicine \& Public Health. 2017;76(2):48-54.

35. O'Leary ST, Brewer SE, Pyrzanowski J, Barnard J, Sevick C, Furniss A, et al. Timing of information-seeking about infant vaccines. The Journal of pediatrics. 2018:203:125-30.e1.

36. Vannice KS, Salmon DA, Shui I, Omer SB, Kissner J, Edwards KM, et al. Attitudes and beliefs of parents concerned about vaccines: impact of timing of immunization information. Pediatrics. 2011;127(Supplement 1):S120-S6.

37. Schurink-van't Klooster T, de Melker $\mathrm{H}$. The National Immunisation Programme in the Netherlands: surveillance and developments in 20182019; 2019.

\section{Publisher's Note}

Springer Nature remains neutral with regard to jurisdictional claims in published maps and institutional affiliations.

Ready to submit your research? Choose BMC and benefit from:

- fast, convenient online submission

- thorough peer review by experienced researchers in your field

- rapid publication on acceptance

- support for research data, including large and complex data types

- gold Open Access which fosters wider collaboration and increased citations

- maximum visibility for your research: over $100 \mathrm{M}$ website views per year

At BMC, research is always in progress.

Learn more biomedcentral.com/submissions 